OPEN ACCESS

Edited by: Junpei Zhou,

Yunnan Normal University, China

Reviewed by: Jing Si,

Beijing Forestry University, China

Xiaoyu Zhang,

Huazhong University of Science and Technology, China

*Correspondence: Jinping Yan jpyan2019@163.com

Specialty section: This article was submitted to

Extreme Microbiology, a section of the journa

Frontiers in Microbiology

Received: 23 October 2019

Accepted: 31 January 2020

Published: 19 February 2020

Citation: Yang $X, W u Y$, Zhang $Y$, Yang $E$, Qu Y, Xu H, Chen Y, Irbis $C$ and Yan $J$ (2020) A Thermo-Active Laccase Isoenzyme From Trametes trogi and Its Potential for Dye Decolorization at High Temperature.

Front. Microbiol. 11:241. doi: 10.3389/fmicb.2020.00241

\title{
A Thermo-Active Laccase Isoenzyme From Trametes trogii and Its Potential for Dye Decolorization at High Temperature
}

Xulei Yang', Yuanyuan Wu' ${ }^{1}$, Yu Zhang ${ }^{1}$, En Yang ${ }^{1}$, Yuan Qu ${ }^{1}$, Huini Xu' ${ }^{1}$, Yuhui Chen ${ }^{2}$, Chagan Irbis ${ }^{1}$ and Jinping Yan ${ }^{1 *}$

\footnotetext{
' Laboratory of Bioconversion, Life Science and Technology College, Kunming University of Science and Technology, Kunming, China, ${ }^{2}$ College of Life Science, Southwest Forest University, Kunming, China
}

A thermo-activation and thermostable laccase isoenzyme (Lac 37 I) produced by Trametes trogii S0301 at $37^{\circ} \mathrm{C}$ was purified to apparent homogeneity by anionic exchange chromatography and sephadex G-75 chromatography, with $12.3 \%$ of yeiled and a specific activity of $343.1 \mathrm{U} \mathrm{mg}^{-1}$. The molecular weight of the purified Lac 37 II was estimated to be approximately $56 \mathrm{kDa}$ in $12 \%$ sodium dodecyl sulfate polyacrylamide gel electrophoresis (SDS-PAGE). The optimal $\mathrm{pH}$ and temperature for the protein was 2.7 and $60^{\circ} \mathrm{C}$, respectively. The purified Lac 37 II showed higher resistance to all tested metal ions and organic solvents except for $\mathrm{Fe}^{2+}$ and $\mathrm{Cd}^{2+}$ at $37^{\circ} \mathrm{C}$ and the activity of the purified Lac 37 was significantly enhanced by $\mathrm{Cu}^{2+}$ at $50 \mathrm{mM}$. The $K_{c a t}, K_{m}$, and $K_{c a t} / K_{m}$ of Lac 37 II were $2.977 \mathrm{~s}^{-1}, 16.1 \mu \mathrm{M}$, and $184.9 \mathrm{~s}^{-1} \mu \mathrm{M}^{-1}$, respecively, in the condition of $\mathrm{pH} 2.7$ and $60^{\circ} \mathrm{C}$ using $\mathrm{ABTS}$ as a substrate. Peptide-mass fingerprinting analysis showed that the Lac 37 II matched to the gene-deduced sequences of Icc3 in T. trogii BAFC 463, other than Lcc1, Lcc 2, and $L C C$ 4. Compared with laccase prepared at $28^{\circ} \mathrm{C}$, the onset of thermo-activation of Lac 37 II activity occurred at $30^{\circ} \mathrm{C}$ with an increase of $10 \%$, and reached its maximum at the temperatures range of $40-60^{\circ} \mathrm{C}$ with an increase of about $40 \%$ of their original activity. Furthermore, Lac 37 II showed the efficient decolorization ability toward triphenylmethane dyes at $60^{\circ} \mathrm{C}$, with decolorization rates of 100 and $99.1 \%$ for $25 \mathrm{mg} \mathrm{L}^{-1}$ malachite and crystal violet in $5 \mathrm{~h}$, respectively, when hydroxybenzotriazole (HBT) was used as a mediator. In conclusion, it is the first time to report a thermoactivation laccase from a thermophilic $T$. trogii strain, which has a better enzyme property and higher decolorization ability among fungal laccases, and it also has a further application prospective in the field of biotechnology.

Keywords: Trametes trogii, thermoactive laccase, thermostable laccase, organic solvent tolerance, dye decolorization 


\section{INTRODUCTION}

Laccases (EC1.10.3.2) are a group of copper-containing polyphenol oxidases that are known as "blue enzymes" for green chemistry due to their ability of oxidize diverse substrates which are similar to lignin or the degradation products of major lignin with molecular oxygen as the final electron acceptor. Due to their high catalytic efficiency and broad substrate specificity, laccases are used in various fields, including biopulping, delignification, biobleaching, environmental pollutants bioremediation, dye decolorization, etc. (Riva, 2006; Bertrand et al., 2017; Younes et al., 2019).

Fungi, especially white rot fungi, are the main laccase producers in nature, and the potential laccase producing strains be utilized in industrial application (Bertrand et al., 2017). Until now, many laccase-producing fungi have been studied and most of the fungi can produce several laccase isozymes (usually more than 10 isoenzymes in the same fungus strain) that showed different kinetic and physicochemical features, which makes it possible to seek new laccase isoenzymes and meets the demands in the industrial applications (Janusz et al., 2013; Zhuo et al., 2016; Zheng et al., 2017). However, the expression of different laccase isoenzymes in the same strain depends on many factors such as the presence of inducers (especially $\mathrm{Cu}^{2+}$ and phenolic compounds), the ratio of carbon and nitrogen, age of the culture and heat shock treatment (Baldrian, 2006; Piscitelli et al., 2011; Janusz et al., 2013; Zhuo et al., 2016; Bertrand et al., 2017). Untill now, few laccase isoenzymes have been isolated and characterized, usually one or two isoenzymes per fungus strain, and most of the laccases isoenzymes isolated so far are found sensitive to extreme conditions of temperature, $\mathrm{pH}$, metal ions, etc. (Janusz et al., 2013; Fonseca et al., 2015; Jaiswal et al., 2015; Othman et al., 2018).

Laccase isoenzymes of thermophilic bacteria and fungi usually possess many attractive properties including high thermal stability, thermo-activation (stimulation of enzyme activity by pre-incubation), and tolerance to organic solvents and ionic concentrations (Hildén et al., 2007; Younes and Sayadi, 2011; Yan et al., 2014a,b), which are demanded biobleaching of pulp and treatment of colored industrial effluents (Wong et al., 2000; Asgher et al., 2008). Previous reports have found that increasing the temperature for laccase production (Tong et al., 2007) and heat shock treatment (Wang et al., 2012) in Trametes strains can induce the expression of different laccase isoenzymes and enhance laccase activity. In our experiment, we observed that T. trogii S0301 strain can grow at $37^{\circ} \mathrm{C}$, but to date, only two native laccase isoenzymes (named Lcc1 and Lcc2) have been purified from this strain, and both of them were obtained from the supernatants cultured at $28^{\circ} \mathrm{C}$ (Colao et al., 2003; Yan et al., 2014a).

Thermo-active enzymes usually are more thermotolerant (Rathi et al., 2000; Hildén et al., 2007; Younes and Sayadi, 2011; Campos et al., 2016). Although the first thermo-active laccase was isolated as early as 1993, few thermo-active laccase isoenzymes have been isolated until now, such as laccase isoenzymes from Fomes sclerodermeus, T. hirsutus, Coliolus zonatu, Marasmius quercophilus, Myceliophtora thermophile, and
Scytalidium thermophilum (Hildén et al., 2007; Younes and Sayadi, 2011). Thermo-activation has been observed in the heterogeneous expressed LCC 3 of T. trogii BAFC 463 in Pichia pastoris (named the recombined LCC3) (Campos et al., 2016), but there are no reports about the thermo-active laccase isoenzyme originated from Trametes until now.

To explore the potential application of laccases in the thermotolerant T. trogii S0301 strain, laccase was obtained from this strain cultured at the temperature of $37^{\circ} \mathrm{C}$. The main objectives of current study were (i) to purify and identify the laccase of $T$. trogii $\mathrm{S} 0301$ produced at $37^{\circ} \mathrm{C}$; (ii) to characterize this laccase isoenzyme; and (iii) to assess the potential application of this laccase isoenzyme by dye decolorization experiments.

\section{MATERIALS AND METHODS}

\section{Chemicals and Strain}

2,2'-Azino bis (3-ethylbenzothiazoline-6-sulfonic acid) (ABTS) and dyes (malachite green, bromophenol blue, and crystal violet) were purchased from Sigma-Aldrich and Merck, respectively. T. trogii S0301 strain employed in the present study was stored in the strain collection of Laboratory of Bioconversion of Life Science and Technology College, Kunming University of Science and Technology, and maintained on a GYP slant at $4^{\circ} \mathrm{C}$ (Yan et al., 2014a,b).

\section{Laccase Production}

Four $1-\mathrm{cm}^{2}$ plugs of the GYP plates incubated at $28^{\circ} \mathrm{C}$ for 4 days were excised with a sterilized cutter and added to each $250 \mathrm{~mL}$ Erlenmeyer flask containing $50 \mathrm{~mL}$ of GYP. After another 5 days incubation, the mycelia were homogenized with glass beads $(0.3 \mathrm{~mm}$ in diameter) and transferred to GYP broth containing $2 \mathrm{mM} \mathrm{Cu}^{2+}$ with $10 \%(\mathrm{v} / \mathrm{v})$ of the seed culture broths. The cultures were incubated in a rotary shaker at $200 \mathrm{rpm}$ at 28 and $37^{\circ} \mathrm{C}$, respectively. Ten-day-old liquid cultures were obtained by centrifuging ( $8000 \mathrm{rpm}, 15 \mathrm{~min}$ ) and the cell-free supernatants were designated as the crude enzyme for the further study.

\section{Laccase Activity and Laccase Thermo-Activation Analysis}

Laccase activity was determined with ABTS as the substrate. The $1.5 \mathrm{~mL}$ substrate solution includes $2 \mathrm{mM}$ ABTS, $100 \mathrm{mM}$ phosphate citrate buffer $(\mathrm{pH} 4.0)$, and $0.1 \mathrm{~mL}$ appropriately diluted crude or purified enzyme was used to determine the activity. The increase in absorbance was monitored at $420 \mathrm{~nm}$ for $3 \mathrm{~min}$. One unit of the enzyme activity was defined as the amount of the enzyme that oxidized $1 \mu \mathrm{mol}$ of the ABTS per minute according to the methods described by Yan et al. (2014b).

For thermo-activation analysis, the enzymes were pretreated at different temperatures $\left(30-80^{\circ} \mathrm{C}\right)$ for $30 \mathrm{~min}$ in $100 \mathrm{mM}$ phosphate citrate buffer ( $\mathrm{pH} 4.0$ ), and then thoroughly cooled on ice for another $30 \mathrm{~min}$. Next, the residual laccase activity was determined. The same amount of enzymes that were not heat-treated but placed on ice as positive control, and the heatdenatured enzymes were served as the negative control. All the assays were carried out in triplicate. 


\section{Laccase Purification}

The purification of the laccase from the crude enzyme of 10-day-old liquid cultures under $37^{\circ} \mathrm{C}$ with the addition of $2 \mathrm{mM} \mathrm{Cu}^{2+}$ was carried out as described in Yang et al. (2011). Briefly, the total protein was precipitated from the crude enzyme using ammonium sulfate (80\% saturation). After dialysis, the enzyme solution was successively treated with a $\mathrm{Q}$ Sepharose ${ }^{\mathrm{TM}}$ ion-exchange chromatography column (GE Healthcare) and a Sephadex G-75 Medium chromatography (Biotopped) column. The fraction containing laccase activity was collected and stored at $-20^{\circ} \mathrm{C}$ for further studies. Sodium dodecyl sulfate-polyacrylamide gel electrophoresis (SDS-PAGE) and native sodium dodecyl sulfatepolyacrylamide gel electrophoresis (Native-PAGE) were carried out according to Shi et al. (2014).

\section{Laccase Identification}

The purified laccase was further separated by Native-PAGE. After the electrophoresis, the gel was stained with citratephosphate buffer (100 mM, pH 4.0) containing $1.0 \mathrm{mM}$ ABTS and the laccase band was collected for further study. MALDITOF/TOF-MS analysis used commercial service provided by Sangon Biotech on 4800 Plus MALDI TOF/TOFTM Analyzer (ABI, Foster City, CA, United States). Mass spectra were obtained in positive ions regime using reflectron. The program $\operatorname{Mascot}^{1}$ was used for protein identification by "peptides fingerprints" and fragmentation spectra. The database NCBI was used for searching homology among proteins of all organisms and fungi with the accuracy mentioned taking into account possible methionine oxidation by atmospheric oxygen and possible modification of cysteine by acrylamide (Younes and Sayadi, 2011; Zheng et al., 2017).

\section{$\mathrm{pH}$ and Temperature Effects on the Purified Laccase and Kinetic Analysis Effects of $\mathrm{pH}$ on Laccase Activity and Laccase Stability}

Characterization analysis of the purified laccase was carried out according to Yan et al. (2014b). To determine the optimum $\mathrm{pH}$ of the purified laccase, the laccase activity was assayed in $100 \mathrm{mM}$ citrate-phosphate buffer adjusted to various $\mathrm{pH}$ values between 2.0 and 8.0, with 1.0 intervals at $30^{\circ} \mathrm{C}$. Effect of $\mathrm{pH}$ on the stability of the purified laccase was studied by verifying the remaining activity after incubating the purified enzyme in the buffer solutions mentioned above at $30^{\circ} \mathrm{C}$ for $36 \mathrm{~h}$. Next, the residual laccase activity of each treatment was compared with the control under the standard assay conditions. All the experiments were performed in triplicate.

\section{Effects of Temperature on Laccase Activity and Laccase Stability}

To determine the optimum $\mathrm{pH}$ of the purified laccase, enzymatic reaction was conducted at temperatures from 30 to $80^{\circ} \mathrm{C}$ with the enzyme in $100 \mathrm{mM}$ citrate-phosphate buffer (pH 4.0), with

${ }^{1}$ http://www.matrixscience.com $10^{\circ} \mathrm{C}$ intervals (Wu et al., 2010). For the thermostability analysis, the half-life at certain temperature $\left(T_{1 / 2}\right)$ was determined after the purified laccase incubated at given temperature $(60,70$, or $\left.75^{\circ} \mathrm{C}\right)$ in phosphate citrate buffer $(100 \mathrm{mM}, \mathrm{pH} 4.0)$ with different time intervals. The residual laccase activity was determined by the standard conditions and the activity of untreated enzyme was used to represent $100 \%$ relative activity. All the experiments were performed in triplicate.

\section{Effects of Metal lons on Laccase Activity}

Metal ions including $\mathrm{Na}^{+}, \mathrm{Fe}^{2+}, \mathrm{Cd}^{2+}, \mathrm{Mn}^{2+}, \mathrm{Zn}^{2+}, \mathrm{Mg}^{2+}$, $\mathrm{Co}^{2+}$, and $\mathrm{Cu}^{2+}$ were added to the standard laccase reaction mixture with a final concentration of 5 and $100 \mathrm{mM}$, and the residual activities were measured under the standard conditions (Shi et al., 2014). The laccase activity of the reaction mixture without metal ions was recorded as $100 \%$. All the experiments were performed in triplicate.

\section{Effects of Various Organic Solvents on Laccase Activity}

To determine the effects of organic solvents on laccase activity, commonly used solvents including methanol, ethanol, and acetonitrile were added to the standard laccase reaction mixture with a final concentration of 1,5 , or $10 \%(\mathrm{v} / \mathrm{v})$ and the residual activities were measured under the standard conditions (Shi et al., 2014). The laccase activity of the reaction mixture without organic solvent was recorded as $100 \%$. All the experiments were performed in triplicate.

\section{Kinetic Study}

The kinetic constants $\left(K_{m}\right.$ and $\left.K_{c a t}\right)$ were determined by using ABTS as substrate in series concentrations ranged from 0 to $2 \mathrm{mM}$ at the optimal condition of the purified laccase in $100 \mathrm{mM}$ citrate-phosphate buffer ( $\mathrm{pH} 2.7)$ at $60^{\circ} \mathrm{C}$. The laccase activity for each substrate concentration was determined three times. The $K_{m}$ and $K_{c a t}$ values were evaluated by the Lineweaver-Burk plot using the Originpro 8 for Windows. All the experiments were performed in triplicate.

\section{Dye Decolorization}

Decolorization experiments were carried out according to Yan et al. $(2014 \mathrm{a}, \mathrm{b})$. The dye decolorization reaction was conducted at $60^{\circ} \mathrm{C}$ with or without the addition of hydroxybenzotriazole (HBT) as a laccase mediator (Younes et al., 2007). Decolorization efficiency was calculated according to the following formula: decolorization (\%) $=A_{0}-A_{t} / A_{0}{ }^{*} 100 \%$, where $A_{0}$ is the initial absorbance and $A_{t}$ is the final absorbance (Younes et al., 2016).

\section{Statistical Analysis}

All data were presented as mean \pm standard deviation (SD) for three replications for each sample. The ANOVA test using the software of Origin pro 8 for Windows used to analyze the experiment data. $P$-value $<0.05$ was considered significant. In addition, all statistical charts were drawn by Origin pro 8 for Windows. Protein sequence alignment was analyzed by DNAMAN software. 


\section{RESULTS AND DISCUSSION}

\section{Thermo-Activation Comparison of the Crude Enzymes From T. trogii S0301 Produced at 28 and $37^{\circ} \mathrm{C}$}

Previously, the thermo-active laccase isoenzymes have been observed in the thermophilic or thermotolerant strains, such as Melanocarpus albomyces, $M$. thermophila, and S. thermophilum (Kiiskinen et al., 2002; Younes and Sayadi, 2011). The original crude laccase activity of the culture filtrate produced at 37 and $28^{\circ} \mathrm{C}$ were 3330.62 and $4861.11 \mathrm{U} \mathrm{L}^{-1}$, respectively. We observed that the crude laccase prepared at $37^{\circ} \mathrm{C}$ showed temperaturedependent activation after 30 min of thermal treatment under the different temperature conditions, while the crude laccase of this strain prepared at $28^{\circ} \mathrm{C}$ did not show obvious thermoactivation under the same experiment conditions (Figure 1A). The onset of thermo-activation of laccase activity occurred at $30^{\circ} \mathrm{C}$ with an increase of $10 \%$, and reached its maximum at the temperatures range of $40-60^{\circ} \mathrm{C}$ with an increase of about $40 \%$ of their original activity (Figure 1A). Further raising temperatures caused the loss of the enzyme activity and the disappearance of thermo-activation. Until now, only two laccase isoenzymes ( $\mathrm{Lcc} 1$ and $\mathrm{Lcc} 2$ ) have been purified from T. trogii strains and neither of them is thermo-active (Yan et al., 2014a; Campos et al., 2016). To further explore whether thermoactivation due to different laccase isoenzymes in the crude enzyme, laccase of this strain produced at $37^{\circ} \mathrm{C}$ was purified and identified.

\section{Laccase Purification}

Using Native-PAGE, two main laccase isoenzymes (Lac 37 I and Lac 37 II) were detected from the crude laccase of T. trogii S0301 which produced at $37^{\circ} \mathrm{C}$ in liquid GYP medium added with $2 \mathrm{mM}$ $\mathrm{Cu}^{2+}$ (Figure 1B). Laccase which secreted in culture medium was purified after ammonium sulfate precipitation. By the first step of the purification, the specific lacase activity of crude laccase was changed from 55.3 to $96.0 \mathrm{U} \mathrm{mg}^{-1}$ (Table 1), followed by the anionic exchange chromatography column and the Sephadex G75 chromatography column kept in tandem. Six protein peaks were obtained by anionic exchange chromatography column, and the first peak showed the laccase activity (111.9 $\mathrm{U} \mathrm{mg}^{-1}$ ) (Table 1). The protein solution from peak 1 was collected and then purified by Sephadex G-75 chromatography column. A final specific activity of $343.06 \mathrm{U} \mathrm{mg}^{-1}$ was achieved. A purification fold of 6.2 and a total enzyme yield of $9.6 \%$ were obtained (Table 1). A single laccase band detected on
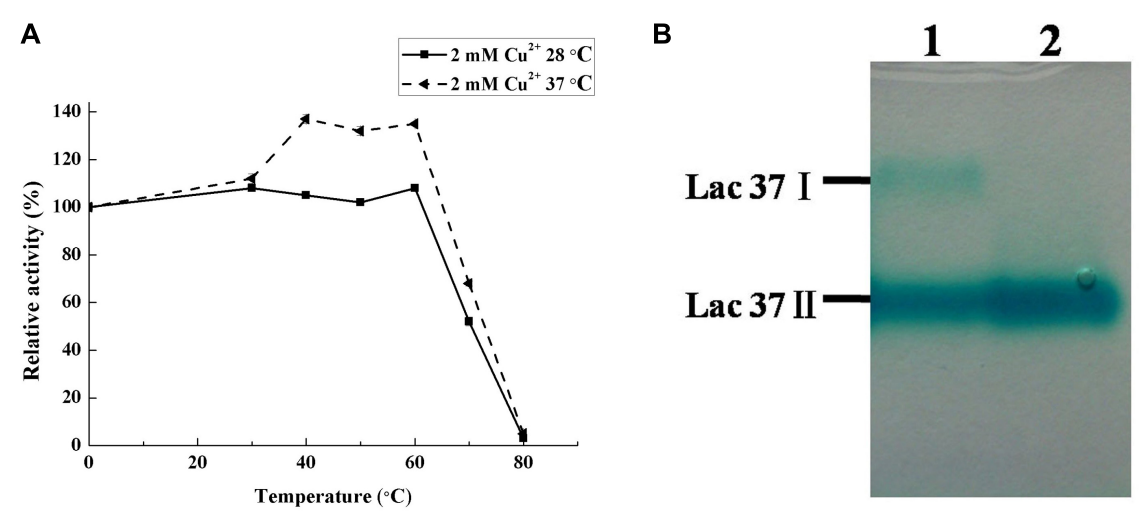

C

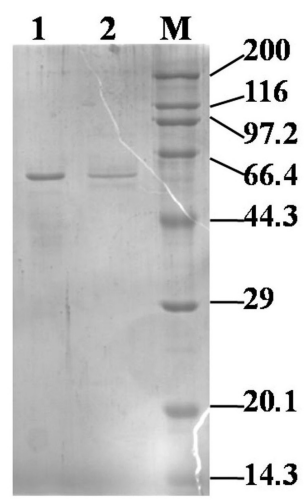

D

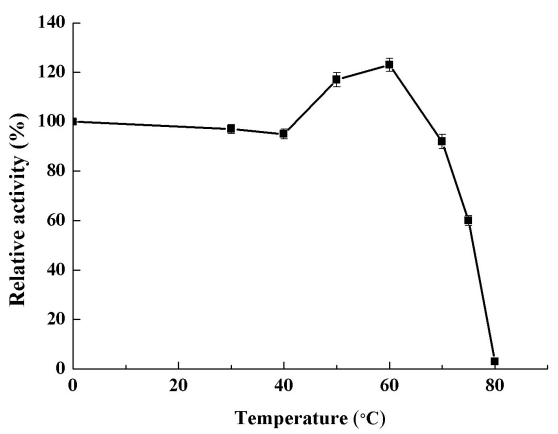

FIGURE 1 | Thermo-activated analysis and purification of laccase from T. trogii S0301. Native-page (B) and SDS-page (C) analysis. Lanes 1 and 2 are the crude and purified laccase produced at $37^{\circ} \mathrm{C}$, respectively. The crude laccase activity of the culture filtrate produced at 37 and $28^{\circ} \mathrm{C}$ were 3330.62 and $4861.11 \mathrm{U} \mathrm{L}-1$ (A). Thermo-activated analysis of purified Lac $37 \|$ from $37^{\circ} \mathrm{C}$, which the original activity is $343.06 \mathrm{U} \mathrm{mg}^{-1}$ (D). Activity is represented as \% relative to the not heat-treated, assigned as 100\%. Data points are the average of triplicate measurements, and the error bars represent the standard deviation. 
TABLE 1 | Summary of laccase purification from T. trogii S0301.

\begin{tabular}{|c|c|c|c|c|c|c|}
\hline Purificatin step & Total volume (mg) & Total activity (U) & Total protein $(\mathrm{mg})$ & Specific activity $\left(\mathrm{U} \mathrm{mg}^{-1}\right)$ & Yield (\%) & Purification fold \\
\hline Crude laccase & 1712 & 3330.62 & 59.6 & 55.31 & 100 & 1 \\
\hline Ammonium sulfate precipitation & 59.57 & 735.03 & 11.137 & 95.99 & 22.07 & 1.74 \\
\hline Anionic exchange chromatography & 4.83 & 440.5 & 4.3 & 111.91 & 13.22 & 2.02 \\
\hline Sephadex G-75 chromatography & 3.4 & 321 & 1.02 & 343.06 & 9.6 & 6.2 \\
\hline
\end{tabular}

TABLE 2 | Comparison of kinetic properties of the purified laccases mainly from strains of Trametes genus.

\begin{tabular}{|c|c|c|c|c|c|c|c|}
\hline Strain & $\begin{array}{l}\text { Specific activity } \\
\qquad\left(\mathrm{U} \mathrm{mg}^{-1}\right)\end{array}$ & $\begin{array}{c}K_{m} \\
(\mu \mathrm{M})\end{array}$ & $\begin{array}{l}K_{c a t} \\
\left(s^{-1}\right)\end{array}$ & $\begin{array}{c}K_{c a t} / K_{m} \\
\left(s^{-1} \mu M^{-1}\right)\end{array}$ & $\begin{array}{l}\text { Optimal condition } \\
\qquad(\mathrm{Tem} / \mathrm{pH})\end{array}$ & $T_{1 / 2}(\min )$ & References \\
\hline $\begin{array}{l}\text { T. trogii S0301 at } 37^{\circ} \mathrm{C} \\
(\text { Lac } 37 \mathrm{II})\end{array}$ & 343.06 & 16.1 & 2977 & 184.96 & $60^{\circ} \mathrm{C} / 2.7$ & $\begin{array}{l}>360\left(\text { at } 60^{\circ} \mathrm{C}\right) \\
120\left(\text { at } 70^{\circ} \mathrm{C}\right)\end{array}$ & This study \\
\hline T. trogii S0301 at $28^{\circ} \mathrm{C}$ & 352.1 & 69 & 7958 & 115 & $45^{\circ} \mathrm{C} / 3.0$ & $180\left(\right.$ at $\left.60^{\circ} \mathrm{C}\right)$ & Yan et al., 2014a \\
\hline T. trogii BAFC $463^{\mathrm{a}}$ & - & - & - & - & $50^{\circ} \mathrm{C} / 4.4$ & $\begin{array}{l}>120\left(\text { at } 60^{\circ} \mathrm{C}\right) \\
<60\left(\text { at } 70^{\circ} \mathrm{C}\right)\end{array}$ & Grassi et al., 2011 \\
\hline T. trogii 201 & 152 & 30 & 3.3 & 0.11 & $-/ 3-3.5$ & - & Garzillo et al., 1998 \\
\hline T. trogii YDHSD & & 7.32 & 260 & 35.6 & $70^{\circ} \mathrm{C} / 2.2$ & $90\left(\right.$ at $\left.60^{\circ} \mathrm{C}\right)$ & Ai et al., 2015 \\
\hline $\begin{array}{l}\text { Recombined Lcc } 1 \text { of } \\
\text { T. trogii }\end{array}$ & 232 & 9.2 & 98.1 & 10.6 & $-/ 2.2$ & $\begin{array}{l}\text { Lost } 90 \% \text { after } 3 \mathrm{~h} \\
\text { at } 60^{\circ} \mathrm{C}, \mathrm{pH} 6\end{array}$ & Colao et al., 2006 \\
\hline $\begin{array}{l}\text { Recombined Lcc } 2 \text { of } \\
\text { T. trogii }\end{array}$ & - & 218 & 5.8 & 0.03 & $-/ 2.5$ & - & Colao et al., 2009 \\
\hline $\begin{array}{l}\text { Recombined Lcc } 3 \text { of } \\
\text { T. trogii BAFC } 463\end{array}$ & - & 250 & 399 & 1.59 & $50^{\circ} \mathrm{C} / 2.7$ & $\begin{array}{l}>180\left(\text { at } 60^{\circ} \mathrm{C}\right) \\
45\left(\text { at } 70^{\circ} \mathrm{C}\right)\end{array}$ & Campos et al., 2016 \\
\hline T. trogi LacA & 11.85 & 54.6 & & & $50^{\circ} \mathrm{C} / 4.5$ & $\begin{array}{l}>240\left(\text { at } 40^{\circ} \mathrm{C}\right) \\
\text { Unstable at } 60^{\circ} \mathrm{C}\end{array}$ & Guan et al., 2011 \\
\hline T. trogi LacB & 4.52 & 17.7 & & & $60^{\circ} \mathrm{C} / 4.0$ & & \\
\hline T. pubescens & 18.543 & 105 & 876 & 8.34 & $50^{\circ} \mathrm{C} / 5.0$ & 120 (at $\left.75^{\circ} \mathrm{C}\right)$ & Si et al., 2013 \\
\hline T. versicolor sdu-4 & 1320 & 47.5 & 284 & 99.7 & $-/ 2.2$ & $132\left(\right.$ at $\left.70^{\circ} \mathrm{C}\right)$ & Zhu et al., 2011 \\
\hline S. thermophilum at $42^{\circ} \mathrm{C}$ & 139.4 & 260 & 1431 & 5.5 & $80^{\circ} \mathrm{C} / 5.0$ & $\begin{array}{l}120\left(\text { at } 65^{\circ} \mathrm{C}\right) \\
90\left(\text { at } 70^{\circ} \mathrm{C}\right)\end{array}$ & Younes et al., 2007 \\
\hline $\begin{array}{l}\text { Cladosporium } \\
\text { cladosporioides at } 42^{\circ} \mathrm{C}\end{array}$ & - & 19.6 & - & - & $40-70^{\circ} \mathrm{C} / 3.5$ & 5 (at $\left.70^{\circ} \mathrm{C}\right)$ & Halaburgi et al., 2011 \\
\hline Echinodontium taxodii 2538 & & 41.4 & - & - & $60^{\circ} \mathrm{C} / 3$ & $>2\left(\right.$ at $\left.50^{\circ} \mathrm{C}\right)$ & Shi et al., 2014 \\
\hline
\end{tabular}

a Laccase was purified but the data were based on the crude laccase. All data in this table were using ABTS as substrate.

Native-PAGE indicated that only Lac 37 II was purified in this study. Lac 37 II showed a single band by SDS-PAGE, with a predicted molecular mass of approximately about $60 \mathrm{kDa}$ (Figure 1C). Most studies showed that the molecular weight of fungal laccase monomer is between 50 and $100 \mathrm{kDa}$, which is consistent with the molecular weight of the purified Lac 37 II (Kunamneni et al., 2008). However, we failed to obtain the other laccase isoenzyme, Lac $37 \mathrm{I}$, in this study. In order to obtain the Lac 37 I, maybe we should change the purification conditions.

The purified Lac 37 II exhibited obvious temperaturedependent activation at temperatures from 40 to $60^{\circ} \mathrm{C}$ and reached its maximum at $60^{\circ} \mathrm{C}$, with an increase of approximately $45 \%$ of the original activity (343.06 $\mathrm{U} \mathrm{mg}^{-1}$ ) (Figure 1D), which was similar to that of the crude enzyme of T. trogii S0301 produced at $37^{\circ} \mathrm{C}$ (Figure 1A). Thus, we suggest that Lac 37 II is the main source of the thermo-active laccase. In our study, the thermo-activation temperature range was agreed with other thermo-cativation laccase isoenzymes, but a highest increasing rate of laccase activity was observed in Lac 37 II, which have approximately $20 \%$ of increase compared to that of Physisporinus rivulosus and S. thermophilum (Hildén et al., 2007; Younes and Sayadi, 2011).

\section{pH and Temperature Effects on Lac 37 II and Kinetic Analysis}

According to the literature, the enzyme properties of many purified and recombined laccases mainly from T. trogii strains and some thermotolerant fungi are summarized in Table 2.

Generally, fungal laccases demonstrate their optimal $\mathrm{pH}$ of 2.0-6.0 using ABTS as substrate (Si et al., 2013; Shi et al., 2014; Ai et al., 2015). Lac 37 II, in this study, exhibited maximal activity at $\mathrm{pH} 2.7$, which is in accordance with the recombined LCC3 and the purified laccases from $T$. trogii S0301 produced at $28^{\circ} \mathrm{C}$ with the optimum of $\mathrm{pH} 2.7$ and 3.0 using ABTS as the substrate, respectively (Figure 2A and Table 2; Yan et al., 2014a; Campos et al., 2016). The original activity of Lac 37 II was stable, maintaining $>80 \%$, after incubation at $\mathrm{pH} 4$ and 5 for $36 \mathrm{~h}$ (Figure 2B). When the $\mathrm{pH}$ was $<3$, the laccase activity was significantly inhibited, with an activity of $18 \%$. These results are in 
A

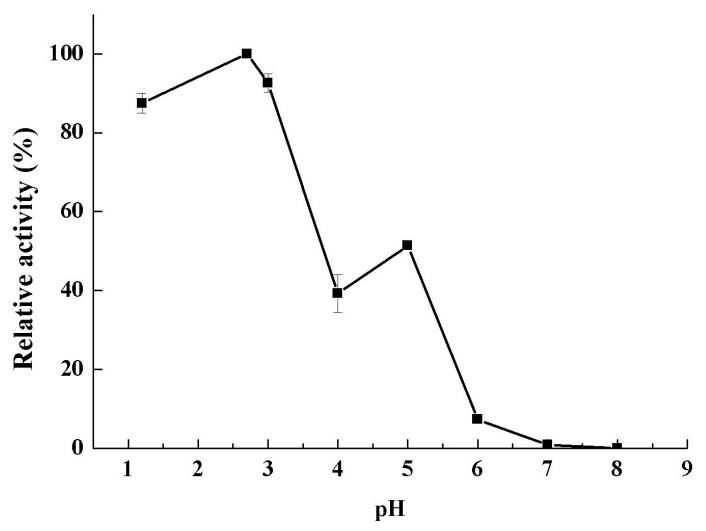

C

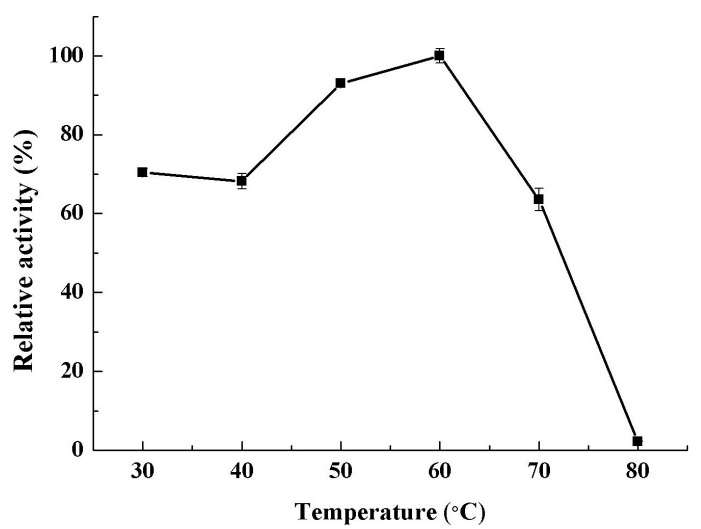

E

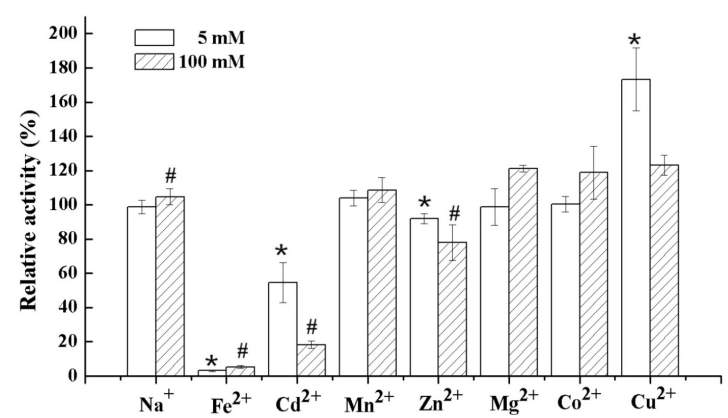

B

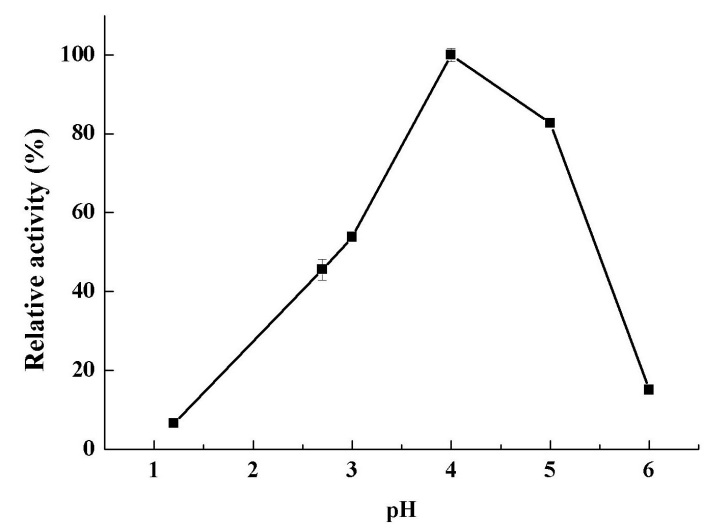

D

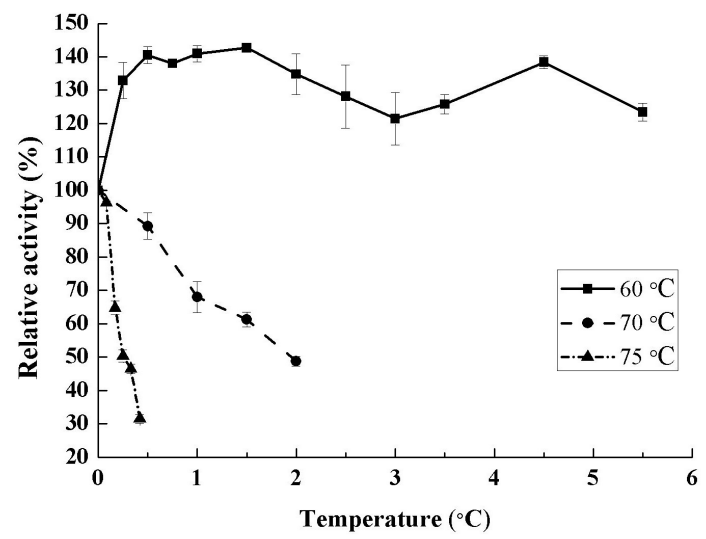

F

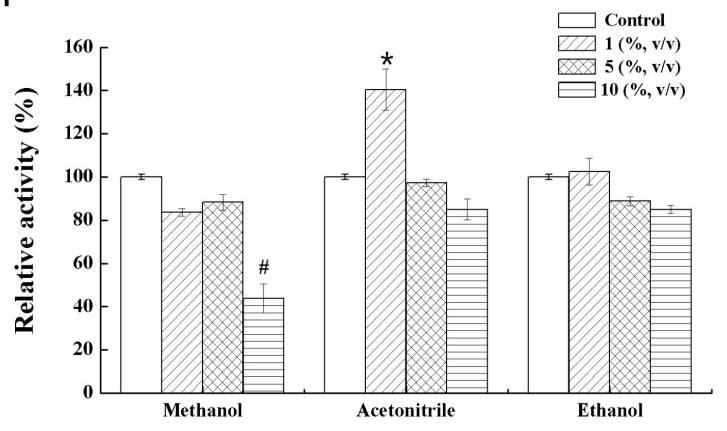

FIGURE 2 | Characterization of Lac $37 \mathrm{ll}$. Effect of $\mathrm{pH}$ on laccase activity (A) and stability for $36 \mathrm{~h}$ (B). Effect of temperature on laccase activity (C) and stability (D). Effect of metal ions (5 or $100 \mathrm{mM}$ ) and organic solvent on laccase activity (E,F). Laccase activity that without heat treatment, metal ions, and organic solvent at pH 4.8 was $343.06 \mathrm{U} / \mathrm{mg}$. Activity is represented as \% relative to laccase activity under the standard conditions, assigned as $100 \%$ for stability studies. The maximum laccase activity was recorded as $100 \%$ for the optimal temperature and $\mathrm{pH}$ studies. The data are presented as means from three independent measurements \pm the standard deviations (indicated by the error bars). ${ }^{*} p$ and ${ }^{\#} p<0.05$, as determined by one-way ANOVA.

accordance with other fungal laccases (Shi et al., 2014; Yan et al., 2014b).

The optimum temperature for Lac 37 II was $60^{\circ} \mathrm{C}$ with ABTS as a substrate, which was higher than that of the recombined LCC3 with the highest laccase activity at $50^{\circ} \mathrm{C}$ (Figure $2 \mathrm{C}$ and Table 2). The half-lives of enzymatic activity at various temperatures $\left(T_{1 / 2}\right)$ at $\mathrm{pH} 4.0$ were $>12 \mathrm{~h}$ at $60^{\circ} \mathrm{C}, 2 \mathrm{~h}$ at $70^{\circ} \mathrm{C}$, and 15 min at $75^{\circ} \mathrm{C}$ with ABTS as the substrate (Figure 2D). Based on the thermostability assays, Lac 37 II in this study exhibited a notable advantage over almost all laccases from the Trametes genus and other sources (Table 2), except for that of $T$. versicolor sdu-4 $\left(T_{1 / 2}\right.$ of $132 \mathrm{~min}$ at $\left.70^{\circ} \mathrm{C}\right)$ (Zhu et al., 2011) and T. pubescens $\left(T_{1 / 2}\right.$ of $120 \mathrm{~min}$ at $\left.75^{\circ} \mathrm{C}\right)$ (Si et al., 2013). 
Kinetic analysis was carried out with ABTS as a substrate at optimal conditions ( $\mathrm{pH} 2.7$ and $60^{\circ} \mathrm{C}$ ). The $K_{m}, K_{c a t}$, and $K_{\text {cat }} / K_{m}$ of Lac 37 II were $16.1 \mu \mathrm{M}, 2977.8 \mathrm{~s}^{-1}$, and $184.9 \mathrm{~s}^{-1} \mu \mathrm{M}^{-1}$, respectively (Table 2 ). Lac 37 II in this study possessed higher thermostability and catalytic efficiency, which makes the laccase isoenzyme have further prospective for the biotechnological applications.

\section{Effect of Metal Ions on Activity of Lac 37 II}

Metal ions are widely distributed in environmental pollutants, and laccases with higher resistance to metal ions are thus attractive (Younes and Sayadi, 2011). In this study, the effects of several metal ions $\left(\mathrm{Na}^{+}, \mathrm{Fe}^{2+}, \mathrm{Cd}^{2+}, \mathrm{Mn}^{2+} \mathrm{Zn}^{2+}, \mathrm{Mg}^{2+}\right.$, $\mathrm{Co}^{2+}$, or $\mathrm{Cu}^{2+}$ ) on Lac 37 II were investigated. The purified Lac 37 II exhibited a high degree of resistance to some metal ions. When the concentration of metal ions was 5 and even $100 \mathrm{mM}$, $\mathrm{Na}^{+}, \mathrm{Mn}^{2+}, \mathrm{Mg}^{2+}, \mathrm{Co}^{2+}$, and $\mathrm{Cu}^{2+}$ had little effects on the laccase activity. However, the other metal ions such as $\mathrm{Fe}^{2+}$, $\mathrm{Cd}^{2+}$, and $\mathrm{Zn}^{2+}$ showed inhibitory effects on the activity of Lac 37 II, especially $\mathrm{Fe}^{2+} . \mathrm{Fe}^{2+}$ completely inhibited the activity of Lac 37 II even at a low concentration $(5 \mathrm{mM})$, and laccase activity decreased to 54.5 and $90.2 \%$ in the presence of $\mathrm{Cd}^{2+}$ and $\mathrm{Zn}^{2+}$ at $5 \mathrm{mM}$, respectively (Figure $2 \mathrm{E}$ ). Some reports have shown that metal ions have some effects on laccase, most of which inhibit laccase activity (Hu et al., 2014; Zhuo et al., 2015). Previous studies have demonstrated that even at low concentrations, $\mathrm{Fe}^{2+}$ (1 or $10 \mathrm{mM}$ ) can strongly inhibit laccase activity in many strains, including Pleurotus ferulae, Pycnoporus sp., T. trogii YDHSD, S. thermophilum, Trametes sp. MA-X01, and T. trogii S0301 (Younes and Sayadi, 2011; Yan et al., 2014a; Ai et al., 2015; Wang et al., 2018). And the purified rLAC-EN3-1 from $P$. pastoris was also sensitive to $\mathrm{Cd}^{2+}$ with relative activities of $62 \%$ at $10 \mathrm{mM}$ and $18 \%$ at $100 \mathrm{mM}$ (Zhuo et al., 2015).

$\mathrm{Cu}^{2+}$, by contrast, obviously enhanced the laccase activity of Lac 37 II with relative activities of 173.3 and $123.1 \%$ at 5 and $100 \mathrm{mM}$, respectively (Figure 2E), which could be due to the role of free copper ions as reducing agents in the solution and reducing the copper center in laccase (Qiao et al., 2017). Those results were in good agreement with the laccase of this strain produced at $28^{\circ} \mathrm{C}$ and of $T$. pubescens with a relative activity of $128 \%$ at $100 \mathrm{mM} \mathrm{Cu}^{2+}$ and $111.3 \%$ at $25 \mathrm{mM} \mathrm{Cu}^{2+}$, respectively (Si et al., 2013; Yan et al., 2014b). Similarly, there was activation of laccase by $10 \mathrm{mM} \mathrm{Cu}^{2+}$ from Sporothrix carnis CPF-05. However, the laccases of Bacillus subtilis cjp3 and T. trogii YDHSD were sensitive to $\mathrm{Cu}^{2+}$ with relative activities of $14 \%$ (Qiao et al., 2017) and $80.9 \%$ at $10 \mathrm{mM}$ (Ai et al., 2015), respectively. Younes and Sayadi (2011) reported that $100 \mathrm{mM} \mathrm{Co}^{2+}$ greatly inhibited laccase activity in $S$. thermophilum and F. fomentarius, compared with relative activities of 100.3 and $77.8 \%$ at $100 \mathrm{mM}$ for laccases from T. trogii S0301 at 37 and $28^{\circ} \mathrm{C}$, respectively.

\section{Effect of Organic Solvents on Activity of Lac 37 II}

Many substrates of laccases are organic pollutants that contain high concentrations of organic solvents used to enhance solubility
(Maté et al., 2010). These will lead to undesirable side reactions of hydrolysis, which is not conducive to thermodynamic equilibrium and difficult to product recovery. The reaction of enzyme catalyst in various organic solvents is greatly limited (Klibanov, 2001). Similarly, the existence of organic solvents is also involved in the application of enzyme membrane immobilization, although it ensures the stability of enzyme to a certain extent (Liu et al., 2019). Thus, fungal laccases with organic cosolvent tolerance have practical uses. The effects of three common solvents (methanol, ethanol, and acetonitrile at a concentration of 1,5 , and $10 \%$ ) on Lac 37 II activity was investigated. The purified Lac 37 II maintained $>80 \%$ of its activity in buffer containing ethanol, even at high concentration $(10 \%, \mathrm{v} / \mathrm{v})$. Among all tested organic solvents, $1 \%(\mathrm{v} / \mathrm{v})$ acetonitrile increased laccase activity by approximately $40.4 \%$, while activity slightly declined to 97.3 and $85.0 \%$ of the control at 5 and $10 \%(\mathrm{v} / \mathrm{v})$, respectively (Figure $2 \mathrm{~F})$. The tolerance of Lac 37 II to acetonitrile and ethanol in this study were similar to that of laccase-like enzyme from the marine sediment samples (Yang et al., 2018). The promotive effect of acetonitrile on laccase activity has been confirmed for the crude laccase of T. trogii LK13 (Yan et al., 2015). In addition, methanol at concentrations ranging from 1 to $5 \%(\mathrm{v} / \mathrm{v})$ slightly lowered activity by $11.7-16.3 \%$, and $10 \%$ ethanol (v/v) led to a $56.1 \%$ loss of activity. Similarly, the catalytic activity of laccase in S. carnis CPF-05 was almost lost when $10 \%$ of the organic solvents added (Olajuyigbe and Fatokun, 2017). In addition, the solvent tolerance of the enzyme is considered to be positively correlated with the thermal stability, which is also in line with the thermoactive and solvent tolerance of Lac 37 II in this study (Rasekh et al., 2014).

\section{Laccase Identification}

Using MALDI-TOF MS, five peptides of Lac 37 II were obtained and the sequences of them were dertermined as follows: KVIAPDGYPR, GPLVVYDPHDPHK, YSFVLEANQPK, ANPNHANFVGFNDGINSAILR, and SAGSSEYNYKNPVQR. These peptides from Lac 37 II accurately matched to the genededuced sequences of $l c c 3$ (GenBank KU055623) in T. trogii BAFC 463, but did not match other laccase isoenzymes of T. trogii strains (Lcc1, Lcc 2, Lcc 4, or the purified laccase of $T$. trogii S0301 at $28^{\circ} \mathrm{C}$ ) (Figure 3). The theoretical protein molecular weight was $56 \mathrm{kDa}$, which is similar to the predicted molecular weight by SDS-PAGE. Previously, lcc3 of $T$. trogii BAFC 463 has been expressed in $P$. pastoris, and the recombined LCC3 showed excellent thermostability and thermo-activation (Campos et al., 2016). The LCC3 was assumed to be due to the thermal stability observed in T. trogii BAFC 463 culture filtrates, but in their study, LCC3 did not purified from the fermentation supernate (Campos et al., 2016). Based on the results of laccase identification, we speculated that Lac 37 II purified in our study is the native LCC3, and it was the third laccase isoenzyme isolated from T. trogii.

We also observed that Lac 37 II showed certain great advantages over the recombined Lcc 3, such as smaller molecular 
LCC 1

LCC2

$\operatorname{LCC} 3$

$\mathrm{LCC} 4$

Lac_28

Lac_37

LCc 1

Lec 2

$\mathrm{LCC} 3$

LCC 4

Lac_28

Lac_37

$\operatorname{LCC} 1$

LCC2

$\mathrm{LCC} 3$

$\mathrm{LCC} 4$

Lac_28

Lac_37

LCC 1

$\mathrm{LCC} 2$

$\operatorname{LCC} 3$

LCC 4

Lac 28

Lac_37

$\mathrm{LCC} 1$

LCC2

$\operatorname{LCC} 3$

LCC 4

Lac_28

Lac_37
MAREQSLITF-TRSLVAS . .VYAAIGPVADLTISNGAVSPDGFSRQAILVNDVFPSPLITGNKGDRFQLNVIDNMTNH MSKFQSLAAF A S LSTGRLAAAA IGPTADLTISNADISPDGFTRAAVVANNQF PGPLITGNKGDTFQLNVIDNLTND MLRTRTRLGN L LLLS LLGTAWGAIGPKTDLHIVNKVIAPDGYPRDTILAGGTF PGPLITGKTGDRFLVDVYDDLTNK MADFRFLHAL VLSFGVL. . SFAAIGPVTDLEITNKNISPDGYERAAVLAGGTFPGALITGKKGDHFQ INVVDDLTNH MARFQSLLTF T SLVAS . VYAAIGPVADLTISNGAVSPDGFSRQA I LVNDVFPSPLITGNKGDRFQLNVIDNMTNH MLHTRTRLGN PLLSLLGTAWGAIGPKTDLH IVNKVIAPDGYPRDTILAGGTF PGPLITGKTGDRFLVDVYDDLTNK

TMLKSTS I HWHGF F HGTNWADGPAFVNQCPISTGHAFLYDFQVPDQAGTFWYHSHLSTQ CDGLRGPIVVYDPQDPH TMLTATT I HWHGFF OKGTNWADGPAFVNQCP I SEGNS F LYDFAAPGQAGTFW HSHLS TQ ECDGLRGVMVVYDPNDPH TMTTPTS I HWHGLFOHNTNWADGAASVTQCPISSGHS F LNFRVP DQAGTFW HSHFGLQ CDGLRGPLVVYDPHDPH TMLKSTS I HWHGFFOKGTNWADGPAFINQCP ISPGNS F LY DFNVP DQAGTFW HSHLSTQ CDGLRGP IVVYDPNDPH TMLKSTS IHWHGFFOHGTNWADGPAFVNQCPISTGHAF LYDEQVPDQAGTFW HSHLSTQECDGLRGPIVVYDPQDPH TMTTPTS I HWHGLFOHNTNWADGAASVTQCP ISSGHSF LYNERVP DQAGTFW HSHFGLQ ECDGLRGPLVVYDPHDPH

\section{$\mathrm{Cu}$ I}

KSLYDVDDDSTVITLADWYHLAAKVGSPVPT . . ADATLINGLGR . SIDTLNADLAVITVTKGKRYRFRLVSLSCDPNH KSLYDVDDESTVITLADWYHTAARLGPREPFG. ADTVLINGLGRFATGDPDAEIAVITVTKGKRYRFRLANISCDPNF KHLYDVDDDSTVITLSDWYHVAAASVPVPPE . SDSTLINGLGR. WGGDPTAELAVITVEHGKRYRFRLLSLSCDPFY ASRYDVDNDDTVITLADWYHVAARLGPAIPFAGADSTLINGKGR. SLGNLDAELSVITVTKGKRYRFRLVS ISCDPNY KSLYDVDDDSTVITLADWYHLAARVGPAIPT . A ADATLINGLGR. S INTLNADLAVITVTKGKRYRFRLVPLSCDPNH KHLYDVDDDSTVITLSDWYHVAAASVPVPPE . . SDSTLINGLGR . WGGDPTAELAVITVEHGKRYRFRLLSLSCDPFY

VFSIDGHSLTVIEADSVNLKPQTVDSIQIFAAQRYSFVLNADQDVGNYWIRALPNSG. TRNFDGGVNSAILRYDGAAP TFS IQGHTMNVIEVDSVNVTPYEVDAIQTFAGQRYSFVLTADQD I DNYWMWA I PNIG. TINTDGGVNSA I LRYDGAAE TFS I DGHDMTI I EADGVNTQPLKVDQLDI LAAQRYSFVLEANQPKGNYWIRANPNHANFVGFNDGINSAILRYKGAPV VFS IDGHDLTVIEADSVNTEPLVVDS IQIFAGQRYSFVLEANKDSDNYWIRANPNFG.VTGFDGGINSAILRYDDAAP TFS IDGHSLTVIEADSVNLKPHTVDS IQIVAAQRYSFVLNADQDVDNYWIRAL PNSG . NTNF DGGVNSA ILRYDGAAP TFS I DGHDMTI IEADGVNTQPLKVDQLDIFAAQRYSFVLEANQPKGNYWIRANPNHANFVGFNDGINSA ILRYKGAPV

VEPTTSQTPSTNPLVESALTTLEGTAAPGSPAPGGVDLALNMAFGFAGGK. . FTINGASFTPPTVPVLLQILSGAQSA VEPDAASPSPSNQLVERNIVPLTDLAAPGNPTVGGVDYALN L PFSF DGAN . FAINGESYTS PSVPVLLQILSGAQTA AEPNTTQTPSKKPLLETNLRPLTKQAVPGKPHPGGANVN IN L LGVDTDRGLFLVNGAPF I P PDVPVLLQILSGNYSA VEPTTTSTS.TKPLKETDLRPLTAMPVPGEAKAGGVDKAIN EAFS FNGSN . FFINGATFAPPAVPVLLQIMSGAQDP VEPTTSQAPSTNPLVESALTTLEGTAAPGSPTPGGVDLALNMAFGFAGGR . FS INGASFTPPTVPVLLQILSGAQSA AEPNTTQTPSKKPLLETNLRPLTKQAVPGKPHPGGANVN IN

QDLLPSGSVYSLPANADIEISLPATAA. APGF FHPFHHG TTEAVVRAGSSTYNYENPVYRDVVSTG . SPGDNVTI PDLLPGGSVYVLPSNATIELSFPMTASNAPGG H HPFHLHG ITFHVVRSAGQTDYNYVNPPQRDTVSVG. .ATGDNVTI QSLLPPGAVYTLPPHQSVEISI PGGVL . . . GGHHPFHLHG HAFSVVRSAGSSEYNYKNPVQRDTVNIG . DSTDNVTI SDLLPSGDIYSLPSNAIIELSFPATVG. APGAAHPFHLHG ITFAVVRSAGSSEYNYDNP IWRDVVNTGTPADGDNVTI QDLLPTGSVYSLPANADIEISLPATTA. APGF HHPFHLHG HAFAVVRSAGSSTYNYENPVYRDVVSTG . SPGDNVTI

Lec4

Lac_-28
$\operatorname{Lac} 37$ QSLLPPGAVYTLPPHQSVEISI PGGVL . . . GGHHPFHLHG HAFSVVRSAGSSEYNYKNPVQRDTVNIG . DSTDNVTI

\section{Cu III}

RFRTDNPGPWFT HCH IDFHIEAGFAVVMAEDIPEVAATNPVPQAWSDLCPTYDALSPDDQ . . . . RFRTDNPGPWFI HCH I DFHI EAGEAVVFAEDTPNVASANKPSAAWEDLCPTYNSVYPNGDNGDAR RFFTDNPGPWET HCH I DFHINAGLAVVFAEDPKDTAFVNPVPSSWKELCPTYYAENP . . . . . RFRTDNPGPWEI HCH I DFHI EAGFAVVMAEDI PNTKSANPPSQAWEDLCPTYNALPVSDQ . . . . RFRTDNPGPWFI HCH I DFHI EAGFAVVMAEDI PDVAATNPVPQAWS DLCPTYDALSPDDQ . . . . RFFTDNPGPWEL HCH IDFHINAGLAVVFAEDPKDTAFVNPVPSSWKELCPTHYAENP . . . . . .

\section{Cu IV}

FIGURE 3 | Multiple amino acid sequences alignments of Lac 37 || with other fungal laccases of $T$. trogii. Four copper-binding conserved domains of typical laccase: Cul (HWHGFFQ), Cull (HSHLSTQ), Culll (HPFHLHG), and CuIV (HCHIDFHL) were boxed in black. The underline showed the internal peptide sequences of Lac 37 II based on the results of MALDI-TOF MS. Lac_28 and Lac_37 were the purified laccase of T. trogii S0301 strain produced at $28^{\circ} \mathrm{C}$ and Lac $37 \|$ at $37^{\circ} \mathrm{C}$, respectively. $\Delta$ indicated the five different amino acids in the gene-deduced sequences between Lac 37 II and Lcc 3.

weight and higher thermostability (Figure 1). In our study, Lac 37 II showed a smaller molecular mass than that of the recombined LCC3 in T. trogii BAFC 463. In addition, the optimum temperature, thermostability, and decolorizing efficiency of Lac 37 II were higher than those of the recombined LCC3 (Table 2).

The possible reasons for those differences are the posttranslational modifications in different hosts (yeasts and 
T. trogii), especially the glycosylation (Maestre-Reyna et al., 2015). Previous studies have proved that the recombined laccases in $P$. pastoris were always hyperglycosylated along with the changes of molecular mass and enzymatic properties, and the mechnism is that the glycosylation profile acts as the regulatory modules for substrate binding and turnover (Younes et al., 2007; Odón et al., 2009; Neha et al., 2012; Maestre-Reyna et al., 2015; Peter, 2016). In addition, we also found that the two amino acids (P13 and P351) of Lac37 II are different from lac3 (L13 and L351) (Figure 3). By predicting the protein structure of Lac $37 \mathrm{II}$, it was found that two prolines were located in the loop region. Suzuki et al. (1987) and Watanabe et al. (1991) considered that the proline may improve the thermal stability of protein by reducing the skeleton entropy of protein unfolding in the proper $\beta$-corner or random curl position. Two mutant lipases improved thermal stability by proline substitution mutagenesis, which were more stable than wild type (Mohammadi et al., 2016). Therefore, the difference of proline may be another factor that affects the thermal stability of Lac 37 II.

\section{Dye Decolorization}

The crude and purified laccase of $T$. trogii S0301 produced at $28^{\circ} \mathrm{C}$ and the recombined LCC 3 all showed high efficiency toward decolorization of triphenylmethane dyes (Grassi et al., 2011; Yan et al., 2014a,b; Campos et al., 2016). Based on these results, two triphenylmethane dyes (malachite green and crystal violet) were chosen in this study to assess the potential application of this laccase isoenzyme. Due to the reasonably good thermostability of the purified laccase, dye decolorization analysis was carried out at $60^{\circ} \mathrm{C}$ in this study.

Without the addition of the laccase mediator, Lac 37 II was less effective against malachite green, and crystal violet, with maximum decolorization of $8.6 \%$ for $25 \mathrm{mg} \mathrm{L}^{-1}$ crystal violet and $16.0 \%$ for $10 \mathrm{mg} \mathrm{L}^{-1}$ malachite green in $15 \mathrm{~h}$, which was similar to the recombined LCC3 (Campos et al., 2016), but much lower

TABLE 3 | Comparison of the decolorization ability of Lac 37 || with other Trametes laccases.

\begin{tabular}{|c|c|c|c|c|c|c|c|}
\hline Strains & $\begin{array}{l}\text { Laccase activity } \\
\qquad\left(\mathrm{U} \mathrm{mL}^{-1}\right)^{a}\end{array}$ & $\begin{array}{l}\text { Dyes } \\
\left(\mathrm{mg} \mathrm{L}^{-1}\right)^{\mathrm{b}}\end{array}$ & Condition & Time (h) & $\begin{array}{c}\text { Decolorization } \\
\text { rate }(\%)\end{array}$ & $\begin{array}{c}\text { Laccase } \\
\text { mediators }^{c}\end{array}$ & References \\
\hline \multirow[t]{5}{*}{ T. trogii S0301 at $37^{\circ} \mathrm{C}$} & 0.25 & $M G(10)$ & $\mathrm{pH} 4.0$ at $60^{\circ} \mathrm{C}$ & 1 & 100 & + & This study \\
\hline & & $M G(10)$ & & 20 & 16 & - & \\
\hline & & MG (25) & & 3 & 100 & + & \\
\hline & & CV (25) & & 5 & 100 & + & \\
\hline & & CV (25) & & 20 & 8.6 & - & \\
\hline \multirow[t]{2}{*}{$\begin{array}{l}\text { Recombined Lcc } 3 \text { of } \\
\text { T. trogii BAFC } 463\end{array}$} & 1 & MG (18) & $\mathrm{pH} 4.5$ at $60^{\circ} \mathrm{C}$ & 24 & 0 & - & $\begin{array}{l}\text { Campos et al., } \\
2016\end{array}$ \\
\hline & & MG (18) & & 2 & 82.8 & + & \\
\hline \multirow[t]{2}{*}{ T. trogii BAFC 463} & 6.5 & MG (8) & & 24 & 98 & + & Grassi et al., 2011 \\
\hline & & MG (8) & & 24 & 25 & - & \\
\hline \multirow[t]{2}{*}{ T. trogii S0301 at $28^{\circ} \mathrm{C}$} & 0.25 & MG (10) & $\mathrm{pH} 4.0$ at $28^{\circ} \mathrm{C}$ & 11 & 83.6 & - & Yan et al., 2014a \\
\hline & & CV (25) & & 11 & 95.7 & - & \\
\hline
\end{tabular}

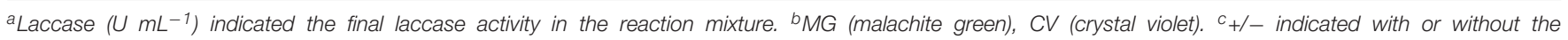
laccase mediators.
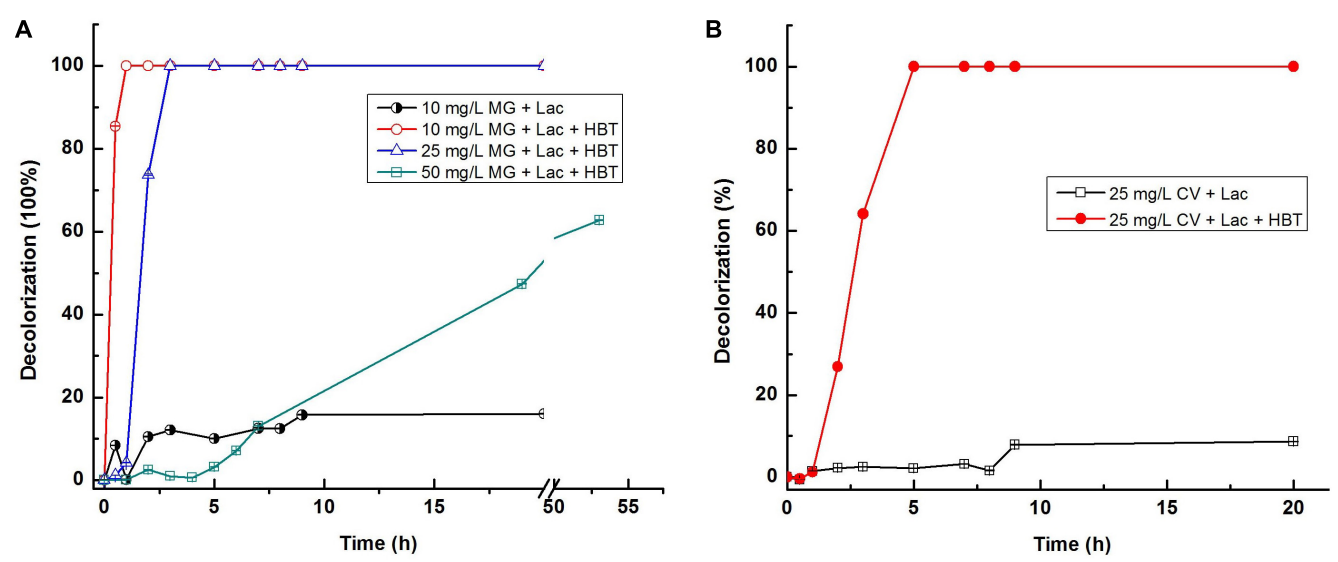

FIGURE 4 | Dye decolorization by Lac 37 II. Reactions were carried out using citrate-phosphate buffer (100 mM, pH 6.0) at $60^{\circ} \mathrm{C}$. MG (malachite green) and CV (crystal violet). The degradation of malachite green (A) and crystal violet (B) were carried out using citrate-phosphate buffer $\left(100 \mathrm{mM}, \mathrm{pH} \mathrm{6.0)}\right.$ at $60^{\circ} \mathrm{C}$. Each value is the mean value \pm standard error of the mean of triplicates. Each value is the mean value \pm standard error of the mean of triplicates. 
than those of the purified laccase from T. trogii S0301 produced at $28^{\circ} \mathrm{C}$ with the maximum decolorization of $95.7 \%$ in $11 \mathrm{~h}$ for all dyes at the same concentration (Yan et al., 2014a) (Table 3).

Previous studies have indicated that the natural or artificial laccase mediators can increase decolorization of dyes by both the purified and crude laccases from many strains, including T. trogii and T. villosa (Grassi et al., 2011; Campos et al., 2016). To confirm whether laccase mediators can improve the decolorization ability of Lac 37 II, 1-HBT was chosed as a laccase mediator. When HBT was added at the concentration of $2 \mathrm{mM}$, the highest decolorization rate of Lac 37 II was detected as approximately $100 \%$ for $10 \mathrm{mg} \mathrm{L}^{-1}$ malachite green within $1 \mathrm{~h}$ (Figure 4A). Lac 37 II efficiently decolorized $25 \mathrm{mg} \mathrm{L}^{-1}$ malachite green, with maximum decolorization of 73.7 and $99.1 \%$ in 2 and $3 \mathrm{~h}$, respectively (Figure 4A). To explore the decolorization ability of Lac 37 II at elevated concentrations of malachite green, $50 \mathrm{mg} \mathrm{L}^{-1}$ malachite green was used. Lac 37 II was able to decolorize with maximum decolorization of 47.3 and $62.7 \%$ in 20 and $53 \mathrm{~h}$, respectively (Figure 4A). HBT also greatly enhanced the decolorization efficiency of Lac 37 II for crystal violet, with decolorization of $26.9,64.1$, and $99.1 \%$ in 2,3 , and $5 \mathrm{~h}$, respectively, while Lac 37 II without HBT showed almost no effect on crystal violet even after $20 \mathrm{~h}$ of incubation, with decolorization of $9.1 \%$ at $20 \mathrm{~h}$ (Figure $4 \mathrm{~B}$ ).

In this study, HBT was chosen as the sole laccase mediator, and a greater increase in decolorization rate was obtained for all tested dyes. However, HBT has been demonstrated as a laccase mediator with a slight or no effect on dyes decolorization (Canas and Camarero, 2010; Campos et al., 2016). Thus, laccase mediators, especially more natural and effective ones, can be optimized in future studies to enhance the decolorization efficiency of Lac 37 II.

\section{CONCLUSION}

In this study, Lac 37 II, a novel native laccase isoenzyme of Trametes trogii S0301 was obtained by incubating this strain at $37^{\circ} \mathrm{C}$, which is higher than the normal cultivation temperature of fungi. By purification and identification, we found that Lac

\section{REFERENCES}

Ai, M. Q., Wang, F. F., and Huang, F. (2015). Purification and characterization of a thermostable laccase from Trametes trogii and its ability in modification of kraft lignin. J. Microbiol. Biotechnol. 25, 1361-1370. doi: 10.4014/jmb.1502.02022

Asgher, M., Bhatti, H. N., Ashraf, M., and Legge, R. L. (2008). Recent developments in biodegradation of industrial pollutants by white rot fungi and their enzyme system. Biodegradation 19, 771-783. doi: 10.1007/s10532-008-9185-3

Baldrian, P. (2006). Fungal laccases - occurrence and properties. FEMS Microbiol. Rev. 30, 215-242. doi: 10.1111/j.1574-4976.2005.00010.x

Bertrand, B., Martínez-Morales, F., and Trejo-Hernández, M. R. (2017). Upgrading laccase production and biochemical properties: strategies and challenges. Biotechnol. Prog. 33, 1015-1034. doi: 10.1002/btpr.2482

Campos, P. A., Levin, L. N., and Wirth, S. A. (2016). Heterologous production, characterization and dye decolorization ability of a novel thermostable laccase isoenzyme from Trametes trogii BAFC 463. Process Biochem. 51, 895-903. doi: $10.1016 /$ j.procbio.2016.03.015
37 II is the third native laccase isoenzyme from T. trogii strains, and it is also the first thermo-active and the more thermostable isoenzyme of Trametes genus strains. With higher thermostability and catalytic efficiency, this laccase isoenzyme can efficiently decolorize triphenylmethane dyes with the addition of a laccase mediator, which makes Lac 37 II have further prospective for biotechnological applications.

\section{DATA AVAILABILITY STATEMENT}

All datasets generated for this study are included in the article/supplementary material.

\section{AUTHOR CONTRIBUTIONS}

$\mathrm{XY}$ has carried out enzyme activity determination, thermal stability analysis, and laccase purification. YW was responsible for the effect of temperature and $\mathrm{pH}$ on the activity and stability of laccase. YZ was responsible for the effect of organic dissolution and metal ions on laccase activity. EY was responsible for the identification of laccase. YQ was responsible for the decolorization of dyes by laccase. HX was responsible for SDSPAGE and Native-PAGE. YC was responsible for the preparation of laccase. CI has revised the original manuscript. JY has conceived the experiment plan, supervised the experiment process, and wrote the original manuscript.

\section{FUNDING}

This work was supported by the National Natural Science Foundation of China (No. 31560036).

\section{ACKNOWLEDGMENTS}

The authors gratefully thank Professor Zhou Tongxin and her team for their valuable help.

Canas, A. I., and Camarero, S. (2010). Laccases and their natural mediators: biotechnological tools for sustainable eco-friendly processes. Biotechnol. Adv. 28, 694-705. doi: 10.1016/j.biotechadv.2010.05.002

Colao, M. C., Caporale, C., Silvestri, F., Ruzzi, M., and Buonocore, V. (2009). Modeling the 3-D structure of a re combinant laccase from Trametes trogii active at a pH close to neutrality. Protein J. 28, 375-383. doi: 10.1007/s10930009-9204-1

Colao, M. C., Garzillo, A. M., Buonocore, V., Schiesser, A., and Ruzzi, M. (2003). Primary structure and transcription analysis of a laccase-encoding gene from the Basidiomycetetrametes trogii. Appl. Microbiol. Biotechnol. 63, 153-158. doi: 10.1007/s00253-003-1429-x

Colao, M. C., Lupino, S., Garzillo, A. M., Buonocore, V., and Ruzzi, M. (2006). Heterologous expression oflcclgene from Trametes trogii in pichia pastoris and characterization of the recombinant enzyme. Microb. Cell Fact. 5, 31-40. doi: 10.1186/1475-2859-5-31

Fonseca, M. I., Farina, J. I., Sadanoski, M. A., D’Errico, R., Villalba, L. L., and Zapata, P. D. (2015). Decolorization of Kraft liquor effluents and biochemical 
characterization of laccases from Phlebia brevispora BAFC 633. Int. Biodeter. Biodegr. 104, 443-451. doi: 10.1016/j.ibiod.2015.07.014

Garzillo, A. M. V., Colao, M. C., Caruso, C., Caporale, C., Celletti, D., and Buonocore, V. (1998). Laccase from the white-rot fungus Trametes trogii. Appl. Microbiol. Biotechnol. 49, 545-551. doi: 10.1007/s002530051211

Grassi, E., Scodeller, P., Filiel, N., Carballo, R., and Levin, L. (2011). Potential of Trametes trogii culture fluids and its purified laccase for the decolorization of different types of recalcitrant dyes without the addition of redox mediators. Int. Biodeter. Biodegr. 65, 635-643. doi: 10.1016/j.ibiod.2011.03.007

Guan, Y., Hua, S., Zhao, X. H., Zhang, Q. H., Zhong, L. J., Zhu, W. W., et al. (2011). Isolation, purification and enzymatic features of laccase from Trametes trogii. J. Microbiol. 31, 64-68. doi: 10.3724/SP.J.1077.2011.00073

Halaburgi, V. M., Sharma, S., Sinha, M., Singh, T. P., and Karegoudar, T. B. (2011). Purification and characterization of a thermostable laccase from the ascomycetes Cladosporium cladosporioides and its applications. Process Biochem. 46, 1146-1152. doi: 10.1016/j.procbio.2011.02.002

Hildén, K., Hakala, T. K., Maijala, P., Lundell, T. K., and Hatakka, A. (2007). Novel thermotolerant laccases produced by the white-rot fungus Physisporinus rivulosus. Appl. Microbiol. Biotechnol. 77, 301-309. doi: 10.1007/s00253-0071155-x

Hu, X., Wang, C., Wang, L., Zhang, R., and Chen, H. (2014). Influence of temperature, $\mathrm{pH}$ and metal ions on guaiacol oxidation of purified laccase from Leptographium qinlingensis. World J. Microbiol. Biotechnol. 30, 1285-1290. doi: 10.1007/s11274-013-1554-3

Jaiswal, N., Pandey, V. P., and Dwivedi, U. N. (2015). Purification of a thermostable alkaline laccase from papaya (Carica papaya) using affinity chromatography. Int. J. Biol. Macromol. 72, 326-332. doi: 10.1016/j.ijbiomac.2014.08.032

Janusz, G., Kucharzyk, K. H., Pawlik, A., Staszczak, M., and Paszczynski, A. J. (2013). Fungal laccase, manganese peroxidase and lignin peroxidase: gene expression and regulation. Enzyme Microb. Technol. 52, 1-12. doi: 10.1016/j. enzmictec.2012.10.003

Kiiskinen, L. L., Viikari, L., and Kruus, K. (2002). Purification and characterisation of a novel laccase from the ascomycete Melanocarpus albomyces. Appl. Microbiol. Biotechnol. 59, 198-204. doi: 10.1007/s00253-002-1012-x

Klibanov, A. M. (2001). Improving enzymes by using them in organic solvents. Nature 409, 241-246. doi: 10.1038/35051719

Kunamneni, A., Plou, F. J., Ballesteros, A., and Alcalde, M. (2008). Laccases and their applications: a patent review. Recent Pat. Biotechnol. 2, 5-12. doi: 10.2174/ 187220808783330965

Liu, C., Saeki, D., Cheng, L., Luo, J., and Matsuyama, H. (2019). Polyketone-based membrane support improves the organic solvent resistance of laccase catalysis. J. Colloid Interface Sci. 544, 230-240. doi: 10.1016/j.jcis.2019.03.003

Maestre-Reyna, M., Liu, W. C., Jeng, W. Y., Lee, C. C., Hsu, C. A., Wen, T. N., et al. (2015). Structural and functional roles of glycosylation in fungal laccase from Lentinus sp. PLoS One 10:e0120601. doi: 10.1371/journal.pone.0120601

Maté, D., García-Burgos, C., García-Ruiz, E., Ballesteros, A. O., Camarero, S., and Alcalde, M. (2010). Laboratory evolution of high-redox potential laccases. Chem. Biol. 17, 1030-1041. doi: 10.1016/j.chembiol.2010.07.010

Mohammadi, M., Sepehrizadeh, Z., Ebrahim-Habibi, A., Shahverdi, A. R., Faramarzi, M. A., and Setayesh, N. (2016). Enhancing activity and thermostability of lipase a from Serratia marcescens by site-directed mutagenesis. Enzyme Microb. Technol. 9, 18-28. doi: 10.1016/j.enzmictec.2016. 07.006

Neha, G., Nora, B., Tenzin, K., Meenu, C., Marion, A. S., and Saroj, M. (2012). Cloning, sequence analysis, expression of Cyathus bulleri laccase in Pichia pastoris and characterization of recombinant laccase. BMC Biotechnol. 12:75. doi: 10.1186/1472-6750-12-75

Odón, V.-V., Palomares, L. A., Dantán-González, E., Ayala-Castro, H. G., Martínez-Anaya, C., Valderrama, B., et al. (2009). The role of $N$-glycosylation on the enzymatic activity of a Pycnoporus sanguineus laccase. Enzyme Microb. Technol. 45, 233-239. doi: 10.1016/j.enzmictec.2009.05.007

Olajuyigbe, F. M., and Fatokun, C. O. (2017). Biochemical characterization of an extremely stable ph-versatile laccase from Sporothrix carnis CPF-05. Int. J. Biol. Macromol. 94, 535-543. doi: 10.1016/j.ijbiomac.2016.10.037

Othman, A. M., Elsayed, M. A., Elshafei, A. M., and Hassan, M. M. (2018). Purification and biochemical characterization of two isolated laccase isoforms from Agaricus bisporus CU13 and their potency in dye decolorization. Int. J. Biol. Macromol. 113, 1142-1148. doi: 10.1016/j.ijbiomac.2018.03.043
Peter, G. (2016). Effects of glycosylation on the enzymatic activity and mechanisms of proteases. Int. J. Mol. Sci. 17:1969. doi: 10.3390/ijms17121969

Piscitelli, A., Giardina, P., Lettera, V., Pezzella, C., Sannia, G., and Faraco, V. (2011). Induction and transcriptional regulation of laccases in fungi. Curr. Genom. 12, 104-112. doi: 10.2174/138920211795564331

Qiao, W., Chu, J., Ding, S., Song, X., and Yu, L. (2017). Characterization of a thermo-alkali-stable laccase from Bacillus subtilis cjp3 and its application in dyes decolorization. J. Environ. Sci. Health A Tox. Hazard. Subst. Environ. Eng. 52, 1-8. doi: 10.1080/10934529.2017.1301747

Rasekh, B., Khajeh, K., Ranjbar, B., Mollania, N., Almasinia, B., and Tirandaz, H. (2014). Protein engineering of laccase to enhance its activity and stability in the presence of organic solvents. Eng. Life Sci. 14, 442-448. doi: 10.1002/elsc. 201300042

Rathi, P., Bradoo, S., Saxena, R. K., and Gupta, R. (2000). A hyper-thermostable, alkaline lipase from Pseudomonas sp. with the property of thermal activation. Biotechnol. Lett. 22, 495-498. doi: 10.1023/a:1005604617440

Riva, S. (2006). Laccases: blue enzymes for green chemistry. Trends Biotechnol. 24, 219-226. doi: 10.1016/j.tibtech.2006.03.006

Shi, L., Yu, H., Dong, T., Kong, W., Ke, M., Ma, F., et al. (2014). Biochemical and molecular characterization of a novel laccase from selective lignin-degrading white-rot fungus Echinodontium taxodii 2538. Process Biochem. 49, 1097-1106. doi: 10.1016/j.procbio.2014.03.028

Si, J., Peng, F., and Cui, B. (2013). Purification, biochemical characterization and dye decolorization capacity of an alkali-resistant and metal-tolerant laccase from Trametes pubescens. Bioresour. Technol. 128, 49-57. doi: 10.1016/j. biortech.2012.10.085

Suzuki, Y., Oishi, K., Nakano, H., and Nagayama, T. (1987). A strong correlation between the increase in number of proline residues and the rise in thermostability of five Bacillus oligo-1,6-glucosidase. Appl. Microbiol. Biotechnol. 26, 546-551. doi: 10.1007/BF00253030

Tong, P. G., Hong, Y. Z., Xiao, Y. Z., Zhang, M., Tu, X. M., and Cui, T. J. (2007). High production of laccase by a new basidiomycete, Trametes sp. Biotechnol. Lett. 29, 295-301. doi: 10.1007/s10529-006-9241-1

Wang, F., Guo, C., Wei, T., Zhang, T., and Liu, C. Z. (2012). Heat shock treatment improves Trametes versicolor laccase production. Appl. Biochem. Biotechnol. 168, 256-265. doi: 10.1007/s12010-012-9769-6

Wang, Q., Ding, L., and Zhu, C. (2018). Characterization of laccase from a novel isolated white-rot fungi Trametes sp. MA-X01 and its potential application in dye decolorization. Biotechnol. Biotechnol. Equip. 32, 1477-1485. doi: 10.1080/ 13102818.2018.1517028

Watanabe, K., Chishiro, K., Kitamura, K., and Suzuki, Y. (1991). Proline residues responsible for thermostability occur with high frequency in the loop regions of an extremely thermostable oligo-1,6-glucosidase from Bacillus thermoglucosidasius kp1006. J. Biol. Chem. 266, 24287-24294. doi: 10.1016/ S1567-4215(03)90519-4

Wong, K. K. Y., Richardson, J. D., and Mansfield, S. D. (2000). Enzymatic treatment of mechanical pulp fibers for improving papermaking properties. Biotechnol. Prog. 16, 1025-1029. doi: 10.1021/bp000064d

Wu, Y. R., Luo, Z. H., Chow, K. K., and Vrijmoed, L. L. P. (2010). Purification and characterization of an extracellular laccase from the anthracene-degrading fungus Fusarium solani MAS2. Bioresour. Technol. 101, 9772-9777. doi: 10. 1016/j.biortech.2010.07.091

Yan, J., Chen, D., Yang, E., Niu, J., Chen, Y., and Chagan, I. (2014a). Purification and characterization of a thermotolerant laccase isoform in Trametes trogii strain and its potential in dye decolorization. Int. Biodeter. Biodegr. 93, 186-194. doi: 10.1016/j.ibiod.2014.06.001

Yan, J., Niu, J. Z., Chen, D. D., Chen, Y. H., and Chagan, I. (2014b). Screening of Trametes strains for efficient decolorization of malachite green at high temperatures and ionic concentrations. Int. Biodeter. Biodegr. 87, 109-115. doi: 10.1016/j.ibiod.2013.11.009

Yan, J. P., Chen, Y. H., Niu, J. Z., Chen, D. D., and Chagan, I. (2015). Laccase produced by a thermotolerant strain of Trametes trogii. Braz. J. Microbiol. 46, 59-65. doi: 10.1590/S1517-838246120130895

Yang, Q., Zhang, M., Zhang, M., Wang, C., Liu, Y., Fan, X., et al. (2018). Characterization of a novel, cold-adapted, and thermostable laccase-like enzyme with high tolerance for organic solvents and salt and potent dye decolorization ability, derived from a marine metagenomic library. Front. Microbiol. 9:2998. doi: 10.3389/fmicb.2018.02998 
Yang, Y., Ma, F., Yu, H., Fan, F., Wan, X., Zhang, X., et al. (2011). Characterization of a laccase gene from the white-rot fungi Trametes sp. 5930 isolated from Shennongjia nature reserve in China and studying on the capability of decolorization of different synthetic dyes. Biochem. Eng. J. 57, 13-22. doi: 10. 1016/j.bej.2011.07.006

Younes, S. B., Cherif, I., Dhouib, A., and Sayadi, S. (2016). Trametes trogii: a biologic powerful tool for dyes decolorization and detoxification. Catal. Lett. 146, 204-211. doi: 10.1007/s10562-015-1629-x

Younes, S. B., Khedher, S. B., Zhang, Y., Geissen, S. U., and Sayadi, S. (2019). Laccase from Scytalidium thermophilum: production improvement, catalytic behavior and detoxifying ability of diclofenac. Catal. Lett. 149, 1833-1844. doi: 10.1007/s10562-019-02771-1

Younes, S. B., Mechichi, T., and Sayadi, S. (2007). Purification and characterization of the laccase secreted by the white rot fungus Perenniporia tephropora and its role in the decolourization of synthetic dyes. J. Appl. Microbiol. 102, 1033-1042.

Younes, S. B., and Sayadi, S. (2011). Purification and characterization of a novel trimeric and thermotolerant laccase produced from the ascomycete Scytalidium thermophilum strain. J. Mol. Catal. B Enzym. 73, 35-42. doi: 10.1016/j.molcatb. 2011.07.014

Zheng, F., An, Q., Meng, G., Wu, X. J., Dai, Y. C., Si, J., et al. (2017). A novel laccase from white rot fungus, Trametes orientalis: purification, characterization, and application. Int. J. Biol. Macromol. 102, 758-770. doi: 10.1016/j.ijbiomac.2017. 04.089
Zhu, Y., Zhang, H., Cao, M., Wei, Z., Huang, F., and Gao, P. (2011). Production of a thermostable metal-tolerant laccase from Trametes versicolor and its application in dye decolorization. Biotechnol. Bioprocess Eng. 16, 1027-1035. doi: 10.1007/ s12257-011-0129-0

Zhuo, R., He, F., Zhang, X., and Yang, Y. (2015). Characterization of a yeast recombinant laccase $\mathrm{rLAC}-\mathrm{EN} 3-1$ and its application in decolorizing synthetic dye with the coexistence of metal ions and organic solvents. Biochem. Eng. J. 93, 63-72. doi: 10.1016/j.bej.2014.09.004

Zhuo, R., Yuan, P., Yang, Y., Zhang, S., Ma, F., and Zhang, X. (2016). Induction of laccase by metal ions and aromatic compounds in Pleurotus ostreatus HAUCC 162 and decolorization of different synthetic dyes by the extracellular laccase. Biochem. Eng. J. 117(Pt B), 62-72. doi: 10.1016/j.bej.2016.09.016

Conflict of Interest: The authors declare that the research was conducted in the absence of any commercial or financial relationships that could be construed as a potential conflict of interest.

Copyright (C) 2020 Yang, Wu, Zhang, Yang, Qu, Xu, Chen, Irbis and Yan. This is an open-access article distributed under the terms of the Creative Commons Attribution License (CC BY). The use, distribution or reproduction in other forums is permitted, provided the original author(s) and the copyright owner(s) are credited and that the original publication in this journal is cited, in accordance with accepted academic practice. No use, distribution or reproduction is permitted which does not comply with these terms. 